\title{
Implantable Unpowered Parylene MEMS Intraocular Pressure Sensor
}

\author{
Po-Jui Chen ${ }^{1}$, Damien C. Rodger ${ }^{1,2}$, Ellis Meng ${ }^{3}$, Mark S. Humayun ${ }^{2}$, and Yu-Chong Tai ${ }^{1}$ \\ ${ }^{1}$ California Institute of Technology \\ 1200 E. California Blvd., Pasadena, CA 91125 USA \\ Tel: +1-626-395-2254, Fax: +1-626-584-9104, E-mail: pjchen@mems.caltech.edu \\ ${ }^{2}$ Keck School of Medicine, University of Southern California \\ Health Science Campus, University of Southern California, Los Angeles, CA 90033 USA \\ ${ }^{3}$ University of Southern California \\ University Park Campus, University of Southern California, Los Angeles, CA 90089 USA
}

\begin{abstract}
This paper presents the first implantable, unpowered, parylene-based micro-electro-mechanical-systems (MEMS) pressure sensor for intraocular pressure (IOP) sensing. From in situ mechanical deformation of the compliant structures, this sensor registers pressure variations without power consumption/transduction. Micromachined high-aspect-ratio thin-walled tubes in different geometric layouts are exploited to obtain a high-sensitivity pressure response. An integrated packaging method has been successfully developed to realize suture-less implantation of the device. In vitro testing results have demonstrated that the IOP sensor can achieve 0.67 degree $/ \mathrm{mmHg}$ angular sensitivity with a spiral-tube design, $3.43 \mu \mathrm{m} / \mathrm{mmHg}$ lateral sensitivity with a long-armed-tube design, and $0.38 \mu \mathrm{m} / \mathrm{mmHg}$ longitudinal sensitivity with a serpentine-tube design. This IOP sensor is designed to be implanted in the anterior chamber of the eye and anchored directly on the iris so that, under incident visible light, the pressure response of the implant can be directly observed from outside the eye, which enables faithful and unpowered IOP monitoring in glaucoma patients.
\end{abstract}

Keywords: Implantable, Intraocular Pressure, Parylene, Pressure Sensor, Unpowered

\section{INTRODUCTION}

Glaucoma is a debilitating disease that results in loss of vision for hundreds of millions of people worldwide. Although measuring intraocular pressure (IOP) is a wellknown method to detect and monitor glaucoma in patients, current tonometry techniques involve only indirect IOP measurements [1][2]. They have difficulties in faithfully recording IOP values inside the eye, and are problematic in deployment for regular (e.g., daily) monitoring of IOP fluctuations and treatment progress. Therefore, a direct and reliable IOP sensing technology is highly needed.

Recently, an all-mechanical unpowered IOP sensing technology has been proposed [3]. By implementing microelectro-mechanical-systems (MEMS) technology, a spiraltube parylene pressure sensor was successfully microfabricated. It demonstrated a linear pressure response without power consumption or external electronic/magnetic/ thermal transduction. Nevertheless, its sensitivity was not high enough to detect pressure variations in the $\mathrm{mmHg}$ range. The size of the sensor was unfavorably large so that implantation in the eye remained a significant problem.
These two factors are the main challenges in using this type of pressure sensor for practical IOP measurement.

In this work, the unpowered IOP sensor paradigm has been further developed in order to provide a solution to these issues. The all-mechanical actuation mechanism is preserved for power-free sensing, while delicate geometric redesign is implemented to achieve higher sensitivity in pressure sensor response. State-of-the-art micromachining techniques are applied to construct high-aspect-ratio rectangular thin-walled tubes with higher flexibility. Furthermore, these smaller IOP sensors are packaged with auxiliary securing components, in an appropriate form factor to enable suture-less implantation. By employing these modifications, our novel IOP sensors permit IOP measurements inside the eye.

\section{DESIGN}

\subsection{IOP Sensor}

The IOP sensing element is a high-aspect-ratio compliant structure inspired by a Bourdon tube, essentially a free- 
standing curved thin-walled tube. They are systematically deformed by a pressure difference applied between the pressure encapsulated in the tube/channel and the ambient pressure, realizing direct pressure measurement through observation of tube deformation. Table 1 shows various geometric layouts of the IOP sensor design to accomplish different in-plane deformation trajectories. In addition to the previous spiral-tube design for angular deformation, a longarmed-tube design that amplifies rotation and a serpentinetube design for linear deformation are extensively developed in this work to facilitate both high-sensitivity pressure response and optimization of device dimensions for sutureless clinical implantation using small outer-diameter needles.

Table 1. Designs of compliant structures for the IOP sensor.

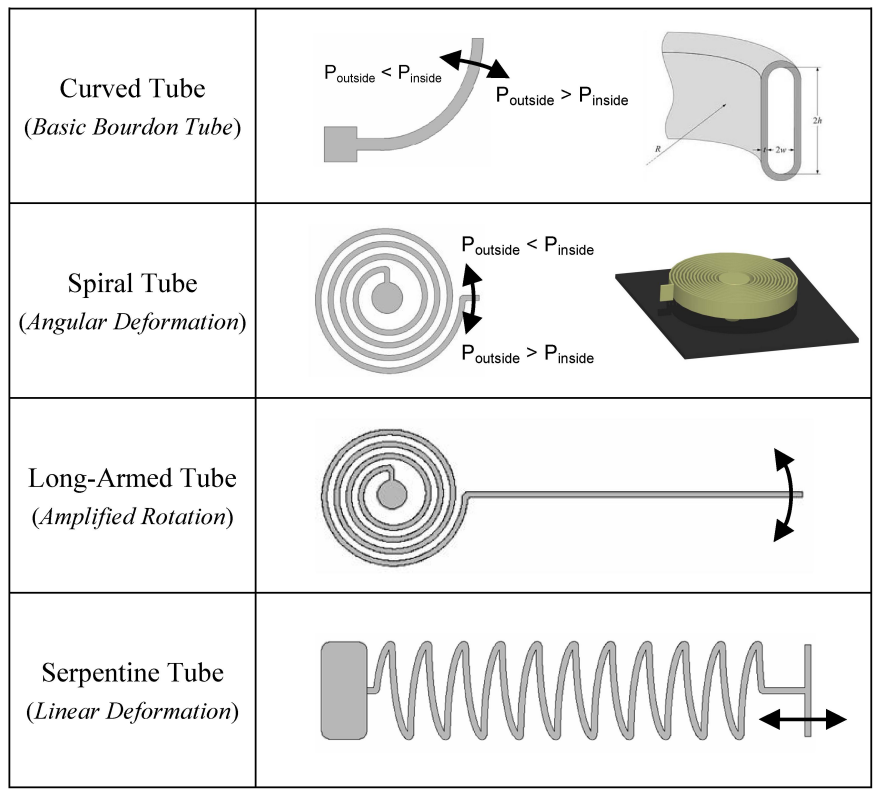

As the main component of the IOP sensor, the thin-walled tubes are designed to be flexible to facilitate pressure responses with high sensitivity. Higher aspect-ratio tubes in a specific shape (e.g. elliptical or rectangular cross-section) are required to obtain larger in-plane deformations and stronger out-of-plane resistance to environmental interference. Parylene C (poly-para-xylene C) is selected as the material for the tube because of its mechanical flexibility (Young's modulus $\sim 4 \mathrm{GPa}$ ), chemical inertness, biocompatibility (USP Class VI grade), and conformality, which enables fabrication of hollow tubes with a single deposition. This material can be utilized to create flexible and implantable biomedical devices.

\subsection{Implantable Device}

Because the IOP sensor is designed to be implanted inside the eye for direct IOP measurement, packaging is necessary to ensure that the IOP sensor is fully functional after implantation. In order to avoid handling and alignment issues during assembly, an integrated packaging method has been developed. By implementing micromachining technology, a fully-packaged device comprising the following components can be fabricated on a monolithic substrate (Fig. 1): (1) A sensor platform to support the IOP sensor; (2) Bottom posts with an optimized "anchoring foot" design to accomplish suture-less anchoring of implant on iris [4]; (3) A perforated flexible parylene membrane to protect the sensor from damage during implantation. With these features, a packaged device can be implanted on the iris so that the sensor faithfully registers IOP variations, which is observable from outside the eye through the cornea and protective parylene membrane.

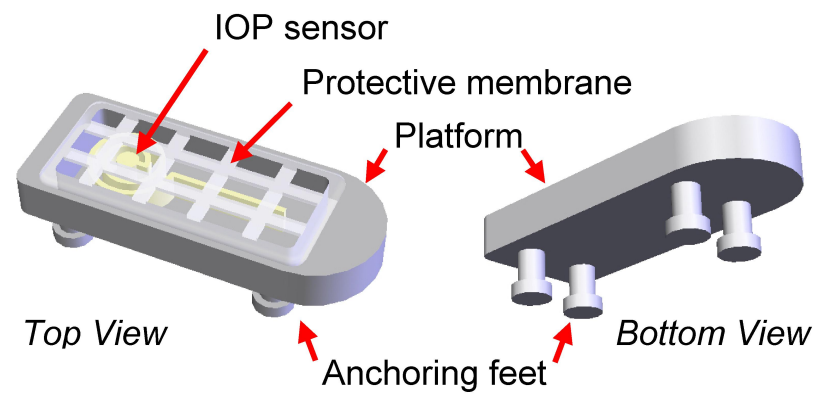

Figure 1. Schematics of fully-packaged implantable device.

\section{FABRICATION}

The device was microfabricated on a monolithic substrate to achieve integrated device packaging. The fabrication process (Fig. 2) started with a $0.75 \mu \mathrm{m}$ wet oxidation on a double-side-polished silicon wafer. After patterning the backside oxide, a backside two-step DRIE was performed to define a $100-\mu \mathrm{m}$-thick sensor platform and $200-\mu \mathrm{m}$-high feet anchors. Fig. 3 shows micrographs of the fabricated feet structures. After that, the frontside oxide was patterned, followed by a two-step complementary dry etching [5] and an 8 - $\mu \mathrm{m}$-thick parylene $\mathrm{C}$ deposition to create $180-\mu \mathrm{m}$-high, $16.5-\mu \mathrm{m}$-wide thin-walled embedded channels with $5-\mu \mathrm{m}-$ thick parylene walls. Channels with an internal channel aspect-ratio of approximately 26 with quasi-rectangular sidewalls (sidewall angle $\sim 90^{\circ} \pm 1^{\circ}$ ) were obtained for flexible mechanical responses. During the parylene deposition to form the channels, the backside structures were simultaneously coated to promote their biocompatibility. The tops of the embedded parylene channels were patterned by oxygen plasma etching to separate them and define the sensor tube features. Subsequent to frontside oxide removal, a sacrificial photoresist layer was spin-coated and the second parylene layer was deposited and patterned to construct a 10$\mu \mathrm{m}$-thick perforated membrane caging the channels with 20 $\mu \mathrm{m}$-high gap. Finally, the photoresist was stripped with acetone and the channels were released by an isotropic $\mathrm{XeF}_{2}$ gaseous silicon etch. Because the silicon surrounding the devices was exposed, the entire fabricated microdevice was separated from the substrate after $\mathrm{XeF}_{2}$ etching without 
dicing, while remaining intact due to protection from parylene-filled trenches indicated in Fig. 2.

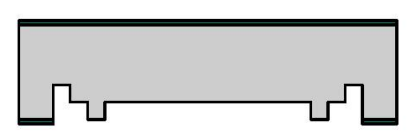

(a) Thermal oxidation

Backside oxide patterning

Backside two-step DRIE

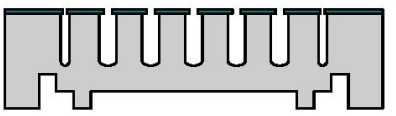

(b) Frontside oxide patterning Complementary HAR trench etching

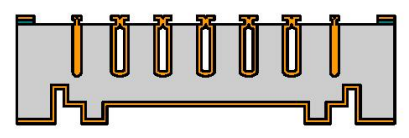

(c) Parylene deposition and patterning Oxide removal

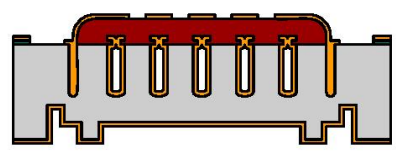

(d) Sacrificial PR coating and patterning Parylene deposition and patterning

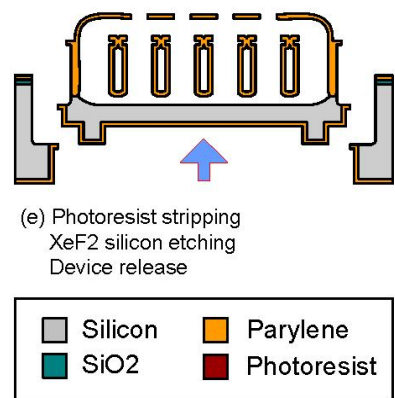

Figure 2. Process flow for device fabrication.

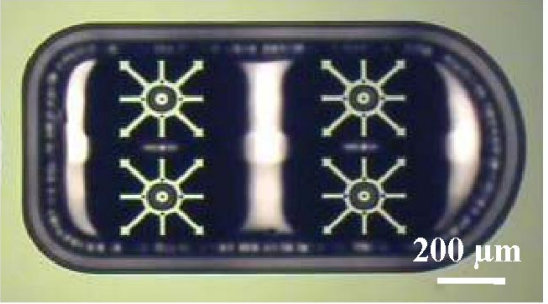

(a)

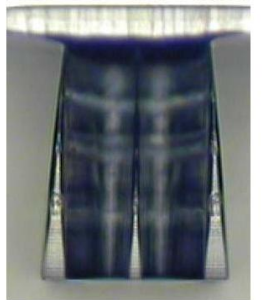

(b)
Figure 3. Backside anchoring foot structures: (a) Top view; (b) Side view (with $200 \mu \mathrm{m}$ height)

Fig. 4 gives micrographs of the devices before the final $\mathrm{XeF}_{2}$ device release. Due to sufficient transparency of parylene C (low light absorption in the visible regime), the embedded IOP sensors in Fig. 4(b) and Fig. 4(c) are clearly observable through the perforated protective membranes.

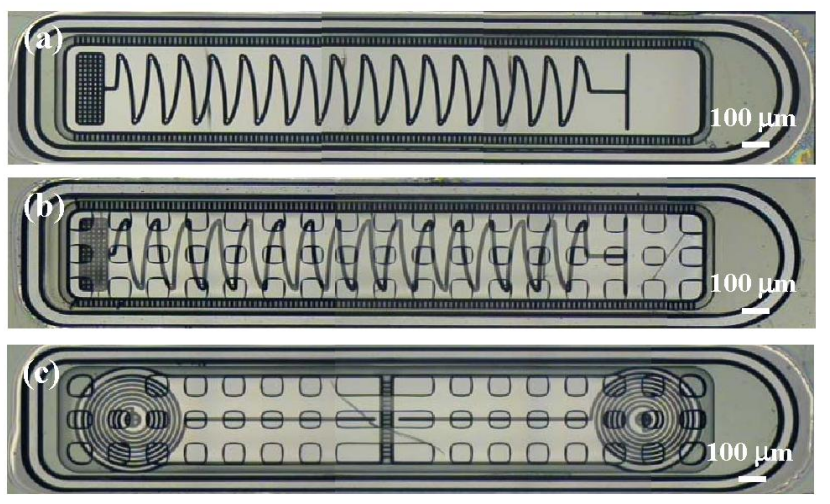

Figure 4. Micrographs of fabricated devices (before $\mathrm{XeF}_{2}$ device release). Sensors in (a) and (b) have serpentine tubes and the sensor in (c) has long-armed tubes.
The final implantable microdevices are shown in Fig. 5. Although the spiral-tube sensors were too large to be implanted using a needle, the long-armed-tube and serpentine-tube sensors were in such a form factor that they could be accommodated inside a 19-gauge needle.

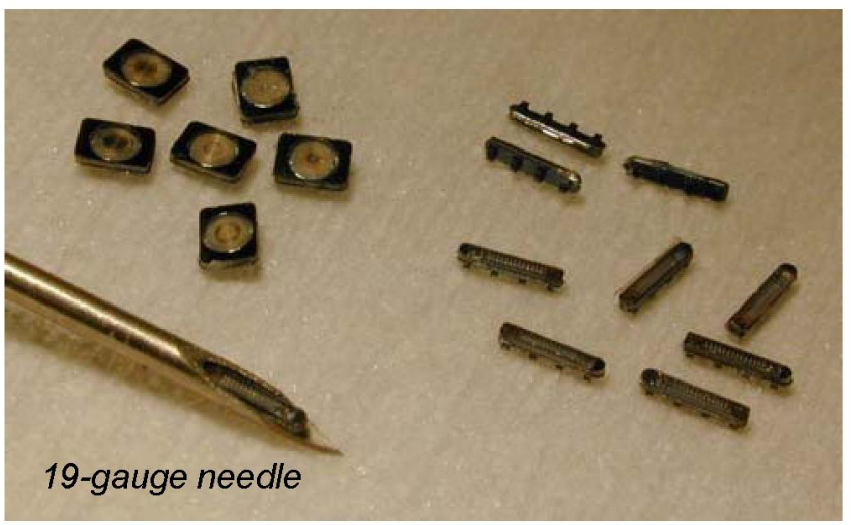

Figure 5. Microfabricated implantable devices.

\section{RESULTS AND DISCUSSION}

Three IOP sensors designs involving a large spiral tube (30 turns, $1.2 \mathrm{~mm} \times 1.2 \mathrm{~mm}$ size), a long-armed spiral tube ( 8 turns, $0.4 \mathrm{~mm} \times 0.4 \mathrm{~mm}$ size with $0.75 \mathrm{~mm}$ pointing tip), and a serpentine tube ( 15 folds, $0.35 \mathrm{~mm} \times 2 \mathrm{~mm}$ size) were tested in vitro in water to characterize their performance. A pressure regulation setup [3] was used to vary the pressure around the sensors. The structural deformation of the sensors with respect to different positive pressures was measured using a stereoscope and compared with numerical readouts from a pressure gauge. Testing results in Fig. 6 and Fig. 7 indicated 0.67 degree/mmHg angular sensitivity with the spiral-tube design, $3.43 \mu \mathrm{m} / \mathrm{mmHg}$ lateral sensitivity with the long-armed-tube design, and $0.38 \mu \mathrm{m} / \mathrm{mmHg}$ longitudinal sensitivity with the serpentine-tube design, all showing a continuous pressure response with high linearity. These data further verified that different geometric layouts of the tube result in different trajectories of tube deformation.

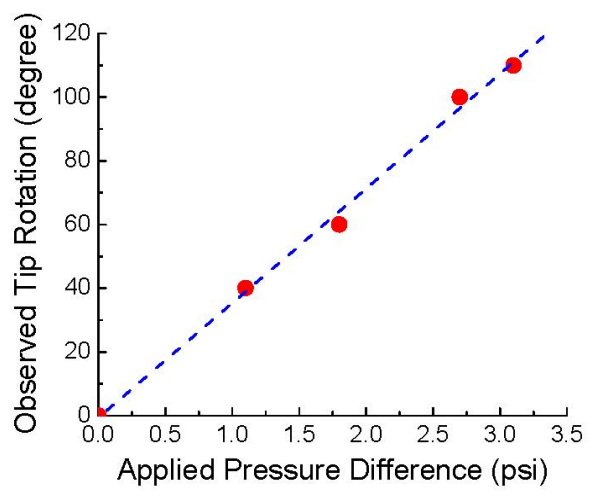

Figure 6. Pressure response of the IOP sensor in the spiraltube configuration (angular trajectory) in water. 


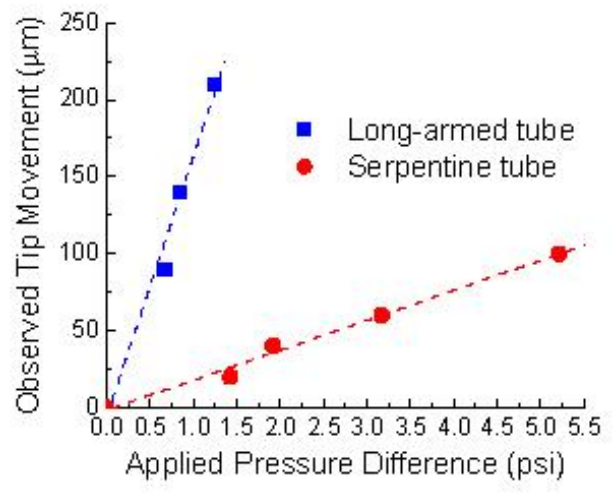

Figure 7. Pressure response of the IOP sensor in the longarmed-tube configuration (lateral trajectory) and in the serpentine-tube configuration (longitudinal trajectory) in water.

Fig. 8 shows a prototype device anchored to human skin. The anchoring mechanism was confirmed to be robust even under vigorous shaking of the finger. This fact implies that the packaged microdevices can be secured on the iris through the use of such tissue anchors upon implantation. Characterization of the devices in the intraocular environment is currently underway to characterize their in vivo performance.

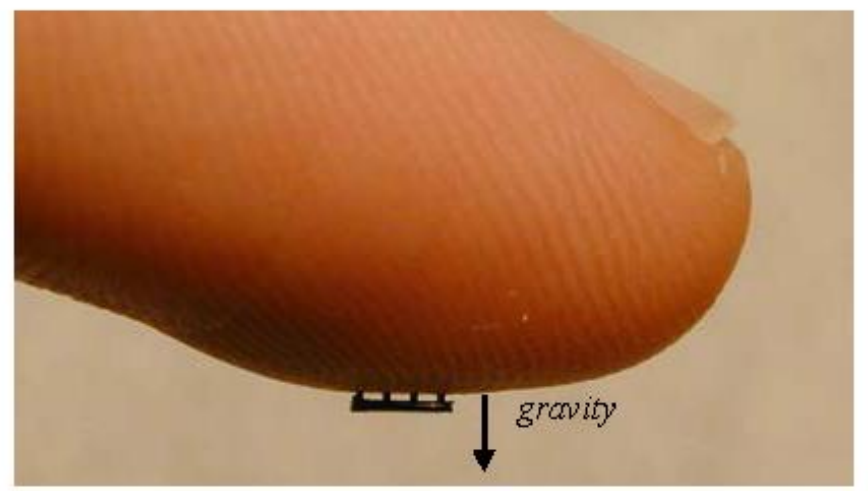

Figure 8. A device prototype with anchoring feet structures attached to human skin

\section{CONCLUSION}

An implantable unpowered parylene-based MEMS pressure sensor has been successfully developed as part of a new paradigm for intraocular pressure (IOP) sensing. Various geometric layouts for micromachined high-aspect-ratio flexible thin-walled tubes including a spiral-tube, a longarmed-tube, and a serpentine-tube design have been implemented to realize pressure sensing with different deformation trajectories. These devices are designed in such a form factor that suture-less implantation through a needle is feasible. Fabrication and packaging of the microdevices have been successfully demonstrated. The microfabricated implantable devices could be accommodated inside a 19 - gauge needle for implantation. Testing results of the on-chip IOP sensors have shown 0.67 degree/mmHg angular sensitivity with the spiral-tube design, $3.43 \mu \mathrm{m} / \mathrm{mmHg}$ lateral sensitivity with the long-armed-tube design, and 0.38 $\mu \mathrm{m} / \mathrm{mmHg}$ longitudinal sensitivity with the serpentine-tube design. These pressure responses indicate that detection of IOP variations in the $\mathrm{mmHg}$ range is possible. A suite of implantation tests is currently underway to further characterize the in vivo performance of the pressure sensors in the intraocular environment. Using the merits of an allmechanical pressure response, the proposed sensing paradigm featuring these implants can provide for direct, faithful, and unpowered IOP monitoring in glaucoma patients.

\section{ACKNOWLEDGEMENTS}

This work was supported in part by the Engineering Research Centers Program of the National Science Foundation under NSF Aw ard Number EEC-0310723. The authors especially thank Mr. Trev or Roper for his fabrication assistance

\section{REFERENCES}

[1] M. Sherwood, in Ophthalmology, M. Yanoff and J. S. Duker, Eds., 2nd ed. St. Louis: Mosby, 2004, pp. 14131473.

[2] H. Cao, in Minimally Invasive Medical Technology, J. G. Webster, Ed. Philadelphia: Institute of Physics Publishing, 2001, pp. 33-45.

[3] P. -J. Chen, D. C. Rodger, M. S. Humayun, and Y. -C. Tai, "Unpowered Spiral-Tube Parylene Pressure Sensor for Intraocular Pressure Sensing," Sensors and Actuators A: Physical, vol. 127, pp. 276-282, 2006.

[4] E. Meng, P.-J. Chen, D. Rodger, Y.-C. Tai, and M. Humayun, "Implantable Parylene MEMS for Glaucoma Therapy," Technical Digest, The Third Annual Intemational IEEE EMBS Special Topic Conference on Microtechnologies in Medicine and Biology (MMMB 2005), Oahu, HI, USA, May 12-15, 2005, pp. 116-119.

[5] P.-J. Chen, D. Rodger, and Y.-C. Tai, "Fully-Dry Fabrication of Monolithic High-Aspect-Ratio Embedded Parylene Microchannels," Technical Digest, The Ninth International Conference on Miniaturized Systems for Chemistry and Life Sciences (MicroTAS 2005), Boston, MA, USA, Oct 9-13, 2005, pp. 181-183. 